\title{
Modeling FACTS for power flow purposes: A common framework
}

\author{
P. Arboleya*, C. Gonzalez-Moran, M. Coto \\ University of Oviedo, Electrical Engineering Departament
}

\begin{abstract}
This paper intends to give a common modeling framework for power flow calculations in power systems with embedded FACTS devices. The proposed method uses the node incidence matrix $(\boldsymbol{\Gamma})$ to avoid the problems derived from the widely used admittance matrix.

The proposed approach is formulated so that the system of differential equations which are the core of the power flow problem, will be kept invariant regardless of the number of embedded FACTS or their location.

As it will be demonstrated, the method provides a very versatile and powerful tool for solving such systems, as it allows for a fast way to change the devices locations, configurations or controls.

All the equations have been stated in a synchronous reference frame $d q$, since it is the most popular reference frame for FACTS control. The main advantage of the proposed problem modeling framework is its simplicity due to the fact that all the equations (both power flow and control equations)
\end{abstract}

\footnotetext{
${ }^{*}$ Corresponding author

Email address: arboleyapablo@uniovi.es, gonzalezmorcristina@uniovi.es, cotomanuel@uniovi.es (P. Arboleya*, C. Gonzalez-Moran, M. Coto)
} 
are defined in a unique reference.

It has to be remarked that what it is proposed in this work, is a common modeling framework, but not an algorithm or solving procedure. The authors tested the proposed framework with the traditional power flow approach and an Optimum Power Flow (OPF) approach.

Keywords: Power flow, FACTS modeling, graph theory, optimal power flow, steady-state modeling

\section{Nomenclature}

\section{Acronyms}

AC Alternating current.

CSM Current source model.

FACTS Flexible AC transmission systems.

GIPFC Generalized interline power flow controller.

HFC Hybrid Flow Controller.

KCL Kirchhoff's current law.

KVL Kirchhoff's voltage law.

PIM Power injection model.

SSSC Static synchronous series compensator.

STATCOM Static synchronous compensator.

UPFC Unified power flow controller.

VSM Voltage source model. 


\section{Matrices}

$\mathbf{R}, \mathbf{L}, \mathbf{X} \quad$ Resistance, inductance and reactance matrices.

I Identity matrix.

M Linear equations matrix.

$\Gamma \quad$ Node incidence matrix.

\section{Parameters}

$\omega \quad$ Pulsation.

\section{Subscripts}

$d, q \quad$ Synchronous reference frame components.

$i, j, k \quad$ Node name or number.

$n_{B} \quad$ Total number of branches (lines).

$n_{N} \quad$ Total number of nodes.

\section{Superscripts}

$B \quad$ Branch or line.

$N \quad$ Node.

se Series.

sh Shunt.

spec Specified.

T Transpossed. 


\section{Variables}

$\begin{array}{ll}e & \text { Injected voltage. } \\ i & \text { Current. } \\ P, Q, S & \text { Active, reactive and apparent powers. } \\ R, L, X & \text { Resistance, inductance and reactance. } \\ v & \text { Voltage. } \\ \theta & \text { Injected voltage angle. }\end{array}$

Vectors

e Injected voltage vector.

i Current vector.

v Voltage vector.

$\mathbf{z} \quad$ Vector of the whole system unknowns.

\section{1. Introduction}

2 Over the years, many methods have been proposed to model and ana3 lyze power systems with embedded FACTS controllers in steady state [1].

${ }_{4}$ This kind of analysis has been applied with different purposes, for instance, 5 sensitivity analysis [2], optimal power system operation based on technical 6 [3-6] or economical considerations [7], sizing of different kind of devices [8], 7 planning and allocation of such devices [9-15], dispatch analysis [16], voltage 8 stability analysis [17] or state estimation [18-21]. 
Basically there exist two kind of models [22]. The first one, the so called decoupled model, where the FACTS devices are substituted by fictitious PQ and/or PV nodes [23], has fallen into disuse in the last years and it has been replaced by the second method known as coupled method, in which the devices are represented in a more intuitive way.

Within the second typo of model, we can distinguish between three different groups. In the first one, the devices are replaced by a current source, so it is called Current Source Model (CSM) [8, 24-27]. The second group is similar to the first one but it uses a voltage source instead, so it is known as Voltage Source Model (VSM) [2, 4, 28, 29]. Finally the Power Injection Model (PIM) substitutes the injected voltage or current sources by power sources, so its main advantage comparing to the other methods is related to the symmetry of the admittance matrix [3, 22, 30-33].

In [34], a hybrid VSM/PIM model for modeling a Hybrid Flow Controller (HFC) was presented, in this case the device was replaced by a power injection in a node and a voltage injection in another one. In [5], a Unified Power Flow Controller (UPFC) is modeled using a hybrid VSM/CIM model. In this case the device is replaced by a shunt current source and a series voltage source.

Regardless of the chosen model, most of the authors use the admittance matrix approach to describe systems with embedded FACTS [16, 29, 33, 3538], being the Newton-Raphson the archetype algorithm for solving these models $[4,16,17,26,29,32,33,35,36,38-41]$. However, the use of the admittance matrix approach presents some serious drawbacks [42]:

- The admittance matrix merges together all parallel lines and shunt 
devices. It is not possible unequivocally go back to the line, transformer or FACTS devices parameters.

- Any change in the system topology or parameters requires rebuilding the whole admittance matrix

For the above mentioned reasons, a group of authors including the signatories of this article, propose the use of the node incidence matrix $\Gamma$ instead of the admittance matrix approach $[9,42-46]$. With the use of $\boldsymbol{\Gamma}$, the information regarding the system, the devices parameters and the topology is separately organized as it will be showed in the next section.

The use of $\boldsymbol{\Gamma}$ is derived from the application of the graph theory to power systems modeling, since this matrix is an algebraic representation of a graph, as it will be explained in the next section. It can be stated then, that the authors assimilate the whole power system into a graph. This is not a new idea, in 1900 Poincare established the principles of algebraic topology introducing the description of a graph using the incidence matrix. Then in 1916, Veblen showed, how the Kirchhoff laws could be formulated by applying Poincare theory [47]. This was just the beginning of the multiple improvements and innovations in the graph theory and its application to the power systems modeling and analysis. The bulk of this improvements took place in the decades of 50s and 60s when the classical topological formulas were modified to fit passive networks containing mutual couplings and active networks (see for instance $[48,49]$ ). Nowadays, the graph theory is still in vogue, but new advances does not lie only in the graph theory itself, but also in its applications to a wide range of different problems like the one that is being described in this paper. 
One common feature to most of the works mentioned until now, is the use of the conventional stationary reference frame in polar or rectangular coordinates. However, an as it was stated in [50], the use of the $d q$ orthogonal synchronous reference frame facilitates the converters control modeling. In the cited case, the authors used the $d q$ reference frame for modeling a Generalized Interline Power Flow Controller (GIPFC).

In this work, the authors propose a common modeling framework for modeling any kind of FACTS device embedded in a power system by using the VSM approach formulated in a $d q$ coordinates reference frame with the use the node incidence matrix $\boldsymbol{\Gamma}$. The proposed model uses a constant topology for describing the whole system, allowing the activation or deactivation of any series or shunt FACTS device at each line or node of the system respectively. The main contributions of the proposed approach are summarized ahead:

- The use of the node incidence matrix $\boldsymbol{\Gamma}$ permits a fast configuration of the devices and simplifies their reallocation in any other part of the system.

- The proposed method keeps the dimension of the system invariant independently of the number of devices, their configuration or their location in the network.

- In most cases, the power converter controls used in FACTS are implemented in an orthogonal-stationary reference frame. So the use of the same reference frame for modeling the rest of the network will unify the formulation of the power system power flow equations and the FACTS devices controls. 
The authors will propose the use of this formulation to be applied in both kind of power flow problems, the traditional power flow problem, where the reference values for the FACTS devices controls are specified, and the optimal power flow problem (OPF), where the reference values of the FACTS controls are non specified unknowns, so they are part of the solution.

The paper is structured as follows. In section II, the common modeling framework using the $d q$ coordinates and $\Gamma$ matrix will be described, demonstrating that different configurations or allocations can be obtained without changing the model core. In section III, the control of different FACTS systems (STATCOM, SSSC and UPFC) will be presented. Then in section IV, the authors will explain how controls are released to solve the problem as an OPF problem. Section $\mathrm{V}$ will present several test cases with both approaches. All these test were validated by means of a power flow commercial software PowerFactory by DigSilent. Finally, in section VI the conclusions will be presented.

\section{FACTS common modeling framework}

In figure 1 the general model of the power system with embedded FACTS is shown. A series FACTS device is placed at each branch and a shunt FACTS device is placed at each node. This is just a section of the whole power system containing two nodes and one line, but each line or bus of the system will be modelled like this section. Doing such model, the the prospects of adding an embedded FACTS device to any node or line in the system are considered. In the last part of this section it will be explained how the model deals with the activation or deactivation of the different embedded 
FACTS devices at different locations without the need of recalculating the whole system topology by means of the node incidence matrix and the graph theory.

Both, series and shunt FACTS devices are modeled as real voltage sources as it can be observed in figure 1. Each branch (or line) has its own impedance, which is represented by $R_{i j}^{B}$ and $L_{i j}^{B}$, plus a real voltage source, representing the series FACTS device, modeled as an ideal voltage source $e_{i j}^{s e}$ and a series $R L$ type impedance, represented by $R_{i j}^{s e}$ and $L_{i j}^{s e}$. Besides the series real voltage source, a shunt real voltage source is placed at each node, representing the shunt connected FACTS device. In this case $e_{i}^{s h}$ and $e_{j}^{s h}$ represent the shunt connected ideal voltage sources at nodes $i$ and $j$ respectively. Both shunt voltage sources have their own $R L$ type impedances, $\left(R_{i}^{s h}, L_{i}^{s h}\right)$ for node $i$ and $\left(R_{j}^{s h}, L_{j}^{s h}\right)$ for node $j$. The current flowing through the line is $i_{i j}^{B}$ and the current through the shunt voltage sources are $i_{i}^{s h}$ and $i_{j}^{s h}$. Finally, the net current injected by the generators and the loads at each node are $i_{i}^{N}$ and $i_{j}^{N}$. The summatories depicted in figure 1 represent the currents flowing from/to other adjacent nodes.

Using the complex vector theory, the Voltage Kirchhoff Law (KVL) in the line and the shunt voltage sources in figure 1 can be expressed as follows [43]:

$$
\begin{aligned}
v_{i j_{d q}}-e_{i j_{d q}}^{s e} & =\left(R_{i j}^{s e}+R_{i j}^{B}\right) \cdot i_{i j_{d q}}^{B}+\left(L_{i j}^{s e}+L_{i j}^{B}\right) \cdot\left(\frac{d}{d t}+j \omega\right) \cdot i_{i j_{d q}}^{B} \\
v_{i_{d q}}-e_{i_{d q}}^{s h} & =R_{i}^{s h} \cdot i_{i_{d q}}^{s h}+L_{i}^{s h} \cdot\left(\frac{d}{d t}+j \omega\right) \cdot i_{i_{d q}}^{s h} \\
v_{j_{d q}}-e_{j_{d q}}^{s h} & =R_{j}^{s h} \cdot j_{j_{d q}}^{s h}+L_{j}^{s h} \cdot\left(\frac{d}{d t}+j \omega\right) \cdot i_{j_{d q}}^{s h}
\end{aligned}
$$


Where: $x_{d q}=x_{d}+j \cdot x_{q}$

$v_{i}, v_{j}$ and $v_{i j}$ are the voltage at nodes $i, j$ and the voltage difference between both of them respectively. Equations (1), (2) and (3) are generic; they serve for either transient or steady-state analysis, and they give us insight to proceed to decouple the system into $d q$ components. In the present case the system will be analyzed in steady state, therefore, the derivative term is null.

Equations (4) and (5) represent the Current Kirchhoff Law (KCL) at nodes $i$ and $j$ :

$$
\begin{aligned}
& \sum_{k=1}^{n_{N}} i_{k i_{d q}}^{B}+i_{i_{d q}}^{N}-i_{i_{d q}}^{s h}-i_{i j_{d q}}^{B}=0 \\
& \sum_{k=1}^{n_{N}} i_{k j_{d q}}^{B}+i_{j_{d q}}^{N}-i_{j_{d q}}^{s h}+i_{i j_{d q}}^{B}=0
\end{aligned}
$$

Separating all voltages and currents into $d$ and $q$ components, equations (1)-(5) can be rewritten in matrix form:

$$
\begin{aligned}
& \left(\begin{array}{c}
v_{i j_{d}} \\
v_{i j_{q}}
\end{array}\right)-\left(\begin{array}{c}
e_{i j_{d}}^{s h} \\
e_{i j_{q}}^{s h}
\end{array}\right)=\ldots \\
& \ldots\left(\begin{array}{cc}
R_{i j}^{s e}+R_{i j}^{B} & -\omega\left(L_{i j}^{s e}+L_{i j}^{B}\right) \\
\omega\left(L_{i j}^{s e}+L_{i j}^{B}\right) & R_{i j}^{s e}+R_{i j}^{B}
\end{array}\right)\left(\begin{array}{c}
i_{i j_{d}}^{B} \\
i_{i j_{q}}^{B}
\end{array}\right) \\
& \left(\begin{array}{c}
v_{i_{d}} \\
v_{i_{q}}
\end{array}\right)-\left(\begin{array}{c}
e_{i_{d}}^{s h} \\
e_{i_{q}}^{s h}
\end{array}\right)=\left(\begin{array}{cc}
R_{i}^{s h} & -\omega L_{i}^{s h} \\
\omega L_{i}^{s h} & R_{i}^{s h}
\end{array}\right) \cdot\left(\begin{array}{c}
i_{i_{d}}^{s h} \\
i_{i_{q}}^{s h}
\end{array}\right) \\
& \left(\begin{array}{c}
v_{j_{d}} \\
v_{j_{q}}
\end{array}\right)-\left(\begin{array}{c}
e_{j_{d}}^{s h} \\
e_{j_{q}}^{s h}
\end{array}\right)=\left(\begin{array}{cc}
R_{j}^{s h} & -\omega L_{j}^{s h} \\
\omega L_{j}^{s h} & R_{j}^{s h}
\end{array}\right) \cdot\left(\begin{array}{c}
i_{j_{d}}^{s h} \\
i_{j_{q}}^{s h}
\end{array}\right)
\end{aligned}
$$




$$
\begin{aligned}
& \left(\begin{array}{l}
\sum_{k=1}^{n_{N}} i_{k i d}^{B} \\
\sum_{k=1}^{n_{N}} i_{k i q}^{B}
\end{array}\right)+\left(\begin{array}{c}
i_{i_{d}}^{N} \\
i_{i_{q}}^{N}
\end{array}\right)-\left(\begin{array}{c}
i_{i_{d}}^{s h} \\
i_{i_{q}}^{s h}
\end{array}\right)-\left(\begin{array}{c}
i_{i_{d}}^{B} \\
i_{i_{q}}^{B}
\end{array}\right)=\left(\begin{array}{l}
0 \\
0
\end{array}\right) \\
& \left(\begin{array}{c}
\sum_{k=1}^{n_{N}} i_{k j_{d}}^{B} \\
\sum_{k=1}^{n_{N}} i_{k j_{q}}^{B}
\end{array}\right)+\left(\begin{array}{c}
i_{j_{d}}^{N} \\
i_{j_{q}}^{N}
\end{array}\right)-\left(\begin{array}{c}
i_{j_{d}}^{s h} \\
i_{j_{q}}^{s h}
\end{array}\right)-\left(\begin{array}{c}
i_{j_{d}}^{B} \\
i_{j_{q}}^{B}
\end{array}\right)=\left(\begin{array}{l}
0 \\
0
\end{array}\right)
\end{aligned}
$$

To extend the proposed formulation to the whole system, the node incidence matrix $\Gamma$ will be employed. For this purposed we are going to consider the whole system as a graph in which each node will represent a vertex. The connections between nodes (branches) will be the graph edges. To construct the matrix $\boldsymbol{\Gamma}$, the edges must be enumerated beginning in the edge whose tail (lower indexed node) is vertex 1 . If there are more than one edge whose tail is vertex 1, they will be numerated in the same order as their head (higher indexed node). Then, the same procedure is applied to the edges whose tail is vertex 2 , and so on. For each pair of connected vertices $(i, j)$ a new row in the $\boldsymbol{\Gamma}$ matrix will be added. The column $i$ will be filled with a 1 , and the column $\mathrm{j}$ will be filled with $\mathrm{a}-1$. Therefore, the $\boldsymbol{\Gamma}$ rows and columns will represent, respectively, the graph edges and vertices. The elements in $\Gamma_{i j}$ are hence given as follows:

- $\Gamma_{i j}=1$ when the tail of the edge $i$ is the vertex $j$.

- $\Gamma_{i j}=-1$ when the head of the edge $i$ is the vertex $j$.

- Otherwise $\Gamma_{i j}=0$. 
Under this assumption equations (6)-(10) can be extended to the whole system as follows:

$$
\begin{gathered}
\boldsymbol{\Gamma}\left(\mathbf{v}_{d}\right)^{T}-\mathbf{I}_{n_{B}}\left(\mathbf{e}_{d}^{s e}\right)^{T}=\mathbf{R}^{s e+B}\left(\mathbf{i}_{d}^{B}\right)-\omega \mathbf{L}^{s e+B}\left(\mathbf{i}_{q}^{B}\right) \\
\boldsymbol{\Gamma}\left(\mathbf{v}_{q}\right)^{T}-\mathbf{I}_{n_{B}}\left(\mathbf{e}_{q}^{s e}\right)^{T}=\mathbf{R}^{s e+B}\left(\mathbf{i}_{q}^{B}\right)+\omega \mathbf{R}^{s e+B}\left(\mathbf{i}_{d}^{B}\right)
\end{gathered}
$$

$$
\begin{aligned}
& \mathbf{I}_{n_{N}}\left(\mathbf{v}_{d}\right)^{T}-\mathbf{I}_{n_{N}}\left(\mathbf{e}_{d}^{s h}\right)^{T}=\mathbf{R}^{s h}\left(\mathbf{i}_{d}^{s h}\right)^{T}-\omega \mathbf{L}^{s h}\left(\mathbf{i}_{q}^{s h}\right)^{T} \\
& \mathbf{I}_{n_{N}}\left(\mathbf{v}_{q}\right)^{T}-\mathbf{I}_{n_{N}}\left(\mathbf{e}_{q}^{s h}\right)^{T}=\mathbf{R}^{s h}\left(\mathbf{i}_{q}^{s h}\right)^{T}+\omega \mathbf{L}^{s h}\left(\mathbf{i}_{d}^{s h}\right)^{T}
\end{aligned}
$$

$$
\begin{aligned}
& (\boldsymbol{\Gamma})^{T}\left(\mathbf{i}_{d}^{B}\right)^{T}-\mathbf{I}_{n_{N}}\left(\mathbf{i}_{d}^{N}\right)^{T}+\mathbf{I}_{n_{N}}\left(\mathbf{i}_{d}^{s h}\right)^{T}=(\mathbf{0})_{n_{N}} \\
& (\boldsymbol{\Gamma})^{T}\left(\mathbf{i}_{q}^{B}\right)^{T}-\mathbf{I}_{n_{N}}\left(\mathbf{i}_{q}^{N}\right)^{T}+\mathbf{I}_{n_{N}}\left(\mathbf{i}_{q}^{s h}\right)^{T}=(\mathbf{0})_{n_{N}}
\end{aligned}
$$

where:

- $\mathbf{R}^{s e+B}$ : is a diagonal matrix of dimensions $\left(n_{B} \times n_{B}\right)$, where $n_{B}$ is the total number of system branches. The $i_{\text {th }}$ term $R_{i}^{s e+B}$ in this matrix represents the sum of the branch resistance and the series voltage source resistance at branch $i$. If there is not a series device allocated at line $i$, then $R_{i}^{s e}$ will be set to zero.

- $\mathbf{L}^{s e+B}$ : is a diagonal matrix of dimensions $\left(n_{B} \times n_{B}\right)$. The $i_{t h}$ term $L_{i}^{s e+B}$ in this matrix represents the sum of the branch inductance and the series voltage source inductance at branch $i\left(L_{i}^{s e}+L_{i}^{B}\right)$. If there is not a series device allocated at line $i$, then $L_{i}^{s e}$ will be set to zero.

- $\mathbf{R}^{s h}$ : is a diagonal matrix of dimensions $\left(n_{N} \times n_{N}\right)$, where $n_{N}$ is the total number of system nodes. The $i_{t h}$ term $R_{i}^{s h}$ in this matrix represents the shunt voltage source resistance at node $i$. If there is not a shunt 
device allocated at node $i$, then $R_{i}^{s h}$ will be set to a value high enough to be considered as infinite.

- $\mathbf{L}^{s h}$ : is a diagonal matrix of dimensions $\left(n_{N} \times n_{N}\right)$. The $i_{t h}$ term $L_{i}^{s h}$ in this matrix represents the shunt voltage source inductance at node $i$. If there is not a shunt device allocated at node $i$, then $L_{i}^{s h}$ will be set to a value high enough to be considered as infinite.

- $\mathbf{v}_{d}, \mathbf{e}_{d}^{s e}, \mathbf{e}_{d}^{s h}$ : are vectors containing respectively the $d$ component of the voltage in the nodes and the series and the shunt injected voltages by all devices. The same definition could be given for $\mathbf{v}_{q}, \mathbf{e}_{q}^{s e}, \mathbf{e}_{q}^{s h}$, but in this case with the $q$ component.

- $\mathbf{i}_{d}^{B}, \mathbf{i}_{d}^{s h}$ : are vectors containing respectively the $d$ component of the current through all lines and through all shunt devices. The same definition could be given for $\mathbf{i}_{q}^{B}, \mathbf{i}_{q}^{s h}$, but in this case with the $q$ component. Equations (11) and (12) represent the KVL in all system lines including the real voltage source in $d$ and $q$ components respectively. Equations (13) and (14) represent the KVL in all shunt connected elements in $d$ and $q$ components respectively. Finally, equations (15) and (16) represent the KCL in all nodes in $d$ and $q$ components. This set of equations (11)-(16) is the linear core of the problem, and it can be stated in a real compact way being summarized in (17):

$$
\mathbf{M z}^{T}=0
$$

Matrix $\mathbf{M}$ is presented in (48) and the vector of unknowns $\mathbf{z}$, containing branch currents and shunt components currents, all the node injected net 
currents, the shunt and series sources voltages and all node voltages, all of them separated into $d$ and $q$ components are shown in equation (18).

$$
\begin{aligned}
& \mathbf{z}=\left[\begin{array}{lllllll}
\mathbf{i}_{d}^{B} & \mathbf{i}_{q}^{B} & \mathbf{i}_{d}^{s h} & \mathbf{i}_{q}^{s h} & \mathbf{i}_{d}^{N} & \mathbf{i}_{q}^{N} & \ldots \\
\ldots & \mathbf{e}_{d}^{s e} & \mathbf{e}_{q}^{s e} & \mathbf{e}_{d}^{s h} & \mathbf{e}_{q}^{s h} & \mathbf{v}_{d} & \mathbf{v}_{q}
\end{array}\right]
\end{aligned}
$$

The total number of unknowns will be $\left(8 n_{N}+4 n_{B}\right)$. Each node will add 4 voltages (the node voltages $v_{d q}$ and the shunt source voltages $e_{d q}^{s h}$ in $d q$ components), and 4 currents (the shunt currents $i_{d q}^{s h}$ and the net injected currents $i_{d q}^{N}$ in $d q$ components). Each branch will add 2 voltages (the series source voltages $e_{d q}^{s e}$ in $d q$ components) and 2 currents (the branch currents $i_{d q}^{B}$ in $d q$ components).

The total number of linear equations in the expression (17) is $\left(4 n_{N}+2 n_{B}\right)$. Besides these linear equations, each node will add two more equations $\left(2 n_{N}\right)$, which can be linear on nonlinear equations depending on the node type. In Table 1, these equations can be observed for different node types. In the case of the slack bus, no equations will be added, but the voltage value will be specified.

We still need to define $\left(2 n_{N}+2 n_{B}\right)$ equations or specify the values of $\left(2 n_{N}+2 n_{B}\right)$ unknowns. It must be remarked that when no shunt or series devices are included in the system, the shunt and series voltages $e_{i j}^{s e}$ and $e_{i}^{s h}$, and the series impedances $R_{i j}^{s e}$ and $L_{i j}^{s e}$ will be set to zero, and all shunt impedances $R_{i}^{s h}, L_{i}^{s h}$ will be set to a value high enough to be considered as an infinite. Even in that case the matrix $M$ will be a regular matrix and the system can be solved. 
When a series device, for instance a SSSC, or a shunt device, like a STATCOM, are located into the system, two new equations must be added. If the device is a combined series/shunt device, as for example a UPFC, four new equations must be considered. In the next section, the equations that need to be added for different kinds of FACTS will be stated as a function of their controls

\section{Specific FACTS models}

In this section, it will be explained how a shunt device (a STATCOM), a series device (a SSSC) and a combined series/shunt device (a UPFC) can be embedded into the system. The authors want to remark that the same procedure could be used to model any other kind of FACTS.

\subsection{STATCOM Modelling}

In the STATCOM case two equations are added by the device; the operating constraint and the control function. The most common case is a STATCOM without energy storage function so the operating constraint will be:

$$
P_{i}^{s h}=e_{i_{d}}^{s h} \cdot i_{i_{d}}^{s h}+e_{i_{q}}^{s h} \cdot i_{i_{q}}^{s h}=0
$$

If an energy storage system is installed, then $P_{i}^{s h}$ must be defined as a specified value or as a function of the network parameters. In the present work, a conventional STATCOM without energy storage will be considered.

The device control will add an extra equation. In this case six different controls were considered, but any other could be implemented. 
Voltage magnitude at local/remote bus:

The magnitude of the voltage at the bus where the shunt device is connected or at any other bus is set to be an specified value $\left|v_{i}\right|^{\text {spec }}$. The device will inject the required reactive power to keep this voltage level. In theory any bus voltage can be controlled but in practice, the voltage control of a remote bus probably won't be possible due to reactive power constraints violation. The equation (20) represents this control.

$$
\sqrt{\left(v_{i_{d}}\right)^{2}+\left(v_{i_{q}}\right)^{2}}=\left|v_{i}\right|^{\text {spec }}
$$

Voltage injection of the STATCOM:

In this case no node voltage is set as an specified value, in this case the control equation (21) fixes the magnitude of the internal voltage of the device $\left|e_{i}^{s h}\right|^{\text {spec }}$. This control is similar to the previous one but without considering the voltage drop derived from the device impedance.

$$
\sqrt{\left(e_{i_{d}}^{s h}\right)^{2}+\left(e_{i_{q}}^{s h}\right)^{2}}=\left|e_{i}^{s h}\right|^{\text {spec }}
$$

Reactive power injection at the local bus:

This direct control specifies the reactive power that the shunt device injects into de grid $\left(Q_{i}^{s h}\right)^{\text {spec }}$, using the expression (22).

$$
e_{i_{q}}^{s h} \cdot i_{i_{d}}^{s h}-e_{i_{d}}^{s h} \cdot i_{i_{q}}^{s h}=\left(Q_{i}^{s h}\right)^{s p e c}
$$

Reactive power flow in a near line:

The reactive power flow in a line connected to the same bus where the shunt device is connected $\left(Q_{j_{k}}^{B}\right)^{\text {spec }}$, is specified in equation (23). 


$$
v_{j_{q}} \cdot i_{k_{d}}^{B}-v_{j_{d}} \cdot i_{k_{q}}^{B}=\left(Q_{j_{k}}^{B}\right)^{\text {spec }}
$$

Active power flow in a near line:

This control is similar to the previous one, but in this case, the equation (24) fixes the active power $\left(P_{j_{k}}^{B}\right)^{s p e c}$, through a line connected to the same bus where the shunt device is installed.

$$
v_{j_{d}} \cdot i_{k_{d}}^{B}+v_{j_{q}} \cdot i_{k_{q}}^{B}=\left(P_{j_{k}}^{B}\right)^{s p e c}
$$

Impedance of the STATCOM:

Expression (25), makes the device behave as if it was a reactance with an specific value $X_{i}^{\text {spec }}$, a negative value would represent a capacitor behaviour.

$$
\frac{e_{i_{q}}^{s h} i_{i_{d}}^{s h}-e_{i_{d}}^{s h} i_{i_{q}}^{s h}}{\sqrt{\left(i_{i_{d}}^{s h}\right)^{2}+\left(i_{i_{q}}^{s h}\right)^{2}}}=X_{i}^{\text {spec }}
$$

\subsection{SSSC Modelling}

Similar to the previous device each series type device will add two equations, the operating constraint and the control equation. For the case of study of a SSSC the operating constraint will be:

$$
P_{i}^{s e}=e_{i_{d}}^{s e} \cdot i_{i_{d}}^{B}+e_{i_{q}}^{s d} \cdot i_{i_{q}}^{B}=0
$$

As it is deducted from (26) the active power injection is forced to zero.

For the SSSC case, four different controls are proposed as follows, but any other control equation could be implemented. 
Voltage magnitude control at a local/remote bus:

Similar to control expressed in (20), the use of this control (see (27)) forces the voltage of one of the nodes where the line containing the series device is connected to be the specified value $\left|v_{i}\right|^{\text {spec }}$.

$$
\sqrt{\left(v_{i_{d}}\right)^{2}+\left(v_{i_{q}}\right)^{2}}=\left|v_{i}\right|^{\text {spec }}
$$

Voltage injection of the SSSC:

The expression (28) specifies the magnitude of series device internal voltage $\left|e_{i}^{s e}\right|^{\text {spec }}$.

$$
\sqrt{\left(e_{i_{d}}^{s e}\right)^{2}+\left(e_{i_{q}}^{s e}\right)^{2}}=\left|e_{i}^{s e}\right|^{\text {spec }}
$$

Reactive power flow:

The active power through the line where the device is connected $\left(Q_{i j}^{B}\right)^{\text {spec }}$, is fixed using the control equation (29).

$$
v_{i_{q}} \cdot i_{i_{d}}^{B}-v_{i_{d}} \cdot i_{i_{q}}^{B}=\left(Q_{i j}^{B}\right)^{\text {spec }}
$$

Active power flow:

The reactive power through the line where the device is connected $\left(P_{i j}^{B}\right)^{\text {spec }}$, is fixed using the control equation (30).

$$
v_{i_{d}} \cdot i_{i_{d}}^{B}+v_{i_{q}} \cdot i_{i_{q}}^{B}=\left(P_{i j}^{B}\right)^{\text {spec }}
$$


Impedance of the SSSC:

In this case, the equation (31), forces the series device to behave as a specified reactance $X_{i}^{\text {spec }}$, negative values would make the device act as a capacitor.

$$
\frac{e_{i_{q}}^{s e} i_{i_{d}}^{B}-e_{i_{d}}^{s e} i_{i_{q}}^{B}}{\sqrt{\left(i_{i_{d}}^{B}\right)^{2}+\left(i_{i_{q}}^{B}\right)^{2}}}=X_{i}^{\text {spec }}
$$

\subsection{UPFC Modelling}

This device is a combination of a series device and a shunt device, so it will add one operating constraint and two control equations. The operating constraints are specified in the equations (32) and (33). They are based on the assumption that there is no energy storage, so the active power consumed by the shunt device has to be provided by the series one or viceverse:

$$
\begin{gathered}
P_{i}^{s h}-P_{i j}^{s e}=0 \\
P_{i}^{s h}-\left(P_{i}^{s h}\right)^{s p e c}=0
\end{gathered}
$$

Five different control types will be proposed (equations (34)-(42)), but any other control will add two equations to the problem.

Active and reactive power flow control in the line where the series device is installed:

This is one of the most typical controls that allows to specify the net active and reactive power flow $\left(\left(P_{i j}^{B}\right)^{\text {spec }}\right.$ and $\left(Q_{i j}^{B}\right)^{\text {spec }}$ respectively), through the line where the series part of the UPFC is connected. Obviously the required active power to be injected by the series device to make such regulation should be extracted from the node where the shunt part of the UPFC is connected, 
fulfilling the expression (32). The proposed control can be implemented adding the expressions (34) and (35).

$$
\begin{aligned}
& v_{i_{d}} \cdot i_{i_{d}}^{B}+v_{i_{q}} \cdot i_{i_{q}}^{B}=\left(P_{i j}^{B}\right)^{\text {spec }} \\
& v_{i_{q}} \cdot i_{i_{d}}^{B}-v_{i_{d}} \cdot i_{i_{q}}^{B}=\left(Q_{i j}^{B}\right)^{\text {spec }}
\end{aligned}
$$

\section{Power flow control by voltage shifting:}

The expression (37) imposes that the voltage magnitud at both sides of the line where the UPFC is installed must be the same. In this case, for obtaining an active power flow matching with the specified value $\left(P_{i j}\right)^{\text {spec }}$ by means of the equation (36), the angles of the voltages at both sides of the line must be shifted.

$$
\begin{gathered}
v_{i_{d}} \cdot i_{i_{d}}^{B}+v_{i_{q}} \cdot i_{i_{q}}^{B}=\left(P_{i j}^{B}\right)^{\text {spec }} \\
\sqrt{\left(v_{i_{d}}\right)^{2}+\left(v_{i_{q}}\right)^{2}}=\sqrt{\left(v_{j_{d}}\right)^{2}+\left(v_{j_{q}}\right)^{2}}
\end{gathered}
$$

Voltage injection control:

This case is very similar to the one described in the expresions (21) or (28), in such cases, the FACTS was only composed by one series device or one shunt connected device. For this reason, only the magnitude of the internal voltage can be controlled. In this case, the FACTS is composed by two devices, one in series and the other one shunt connected. For this reason we can control the internal voltage of one of them in magnitude and angle. The expressions (38) and (39) fixed the magnitude and the angle of the series device internal voltage, $\left(\left|e_{i}^{\text {se }}\right|^{\text {spec }}\right.$ and $\theta^{\text {spec }}$ respectively). 


$$
\begin{gathered}
\sqrt{\left(e_{i_{d}}^{s e}\right)^{2}+\left(e_{i_{q}}^{s e}\right)^{2}}=\left|e_{i}^{s e}\right|^{\text {spec }} \\
\arctan \left(\frac{e_{i_{q}}^{s e}}{e_{i_{d}}^{s e}}\right)=\theta^{\text {spec }}
\end{gathered}
$$

Phase shifting regulation:

This control is similar to the one expressed in equations (36) and (37). In such case the voltage magnitude at both sides of the line was the same and the angle should be shifted a required amount to obtain the desired active power flow. In this case, the expressions (40) and (41) indicate that the voltage magnitud at both sides of the line where the series part of the UPFC is connected must be the same, but the shift angle between the two voltages $\theta^{\text {spec }}$ is specified also, so now the active power flow is an output of the problem.

$$
\begin{gathered}
\sqrt{\left(v_{i_{d}}\right)^{2}+\left(v_{i_{q}}\right)^{2}}=\sqrt{\left(v_{j_{d}}\right)^{2}+\left(v_{j_{q}}\right)^{2}} \\
\arctan \left(\frac{v_{i_{q}}}{v_{i_{d}}}\right)-\arctan \left(\frac{v_{j_{q}}}{v_{j_{d}}}\right)=\theta^{\text {spec }}
\end{gathered}
$$

Line impedance compensation:

This last case, makes the line to behave as a given impedance, the resistive part $R_{i}^{\text {spec }}$ and the inductive part $X_{i}^{\text {spec }}$ can be specified, a negative value of this last makes the line behave as a capacitor. The equations to run this control are (42) and (43). 


$$
\begin{gathered}
\frac{Q_{i}^{s e}}{\left(I_{i}^{B}\right)^{2}}=\frac{e_{i_{q}}^{s e} i_{i_{d}}^{B}-e_{i_{d}}^{s e} i_{i_{q}}^{B}}{\left(i_{i_{d}}^{B}\right)^{2}+\left(i_{i_{q}}^{B}\right)^{2}}=X_{i}^{\text {spec }} \\
\frac{P_{i}^{s e}}{\left(I_{i}^{B}\right)^{2}}=\frac{e_{i_{d}}^{s e} i_{i_{d}}^{B}+e_{i_{q}}^{s e} i_{i_{q}}^{B}}{\left(i_{i_{d}}^{B}\right)^{2}+\left(i_{i_{q}}^{B}\right)^{2}}=R_{i}^{\text {spec }}
\end{gathered}
$$

In section IV an Optimal Power Flow problem is employed to solve the defined system of equations.

\section{OPF Approach}

For the OPF approach, the authors will use just the equations describing the operating constraints. These equations were defined for the STATCOM, SSSC and UPFC cases in (19), (26) and ((32)-(33)) respectively.

The control equations will be omitted in order to give the system the required degrees of freedom to minimize the target function. In the case of the UPFC we also deactivate the operating constraint given in (32), allowing the problem to calculate optimum energy transfer between the series and the shunt device. The use of a constrained OPF problem is recommended in this case . The most usual constraints in this kind of problems are the maximum and the minimum node voltages, the maximum and the minimum active and reactive powers injected by the generators and the maximum apparent line powers.

For FACTS devices the constraints included in the present OPF approach have to do with the maximum and minimum injected voltage and current as it is stated in the next equations. 


$$
\begin{aligned}
& \left|e_{i}^{s e}\right|^{\text {min }} \leq \sqrt{\left(e_{i_{d}}^{s e}\right)^{2}+\left(e_{i_{q}}^{s e}\right)^{2}} \leq\left|e_{i}^{s e}\right|^{\text {max }} \\
& \left|i_{i}^{s e}\right|^{\text {min }} \leq \sqrt{\left(i_{i_{d}}^{B}\right)^{2}+\left(i_{i_{q}}^{B}\right)^{2}} \leq\left|i_{i}^{s e}\right|^{\text {max }} \\
& \left|e_{i}^{s h}\right|^{\min } \leq \sqrt{\left(e_{i_{d}}^{s h}\right)^{2}+\left(e_{i_{q}}^{s h}\right)^{2}} \leq\left|e_{i}^{s h}\right|^{\max } \\
& \left|i_{i}^{s h}\right|^{\min } \leq \sqrt{\left(i_{i_{d}}^{s h}\right)^{2}+\left(i_{i_{q}}^{s h}\right)^{2}} \leq\left|i_{i}^{s h}\right|^{\max }
\end{aligned}
$$

\section{Test Cases}

To test the proposed formulation, the IEEE 14 node system standard [51] has been chosen (see figure 2). The authors adopted all specified data in the standard excluding the loads, that have been increased in $250 \%$ in order to obtain a lower voltage profile and an overloaded scenario. All the calculations have been carried out in per unit (pu.) system.

Under these assumptions, the obtained results for the base case with no embedded FACTS can be observed in Tables 3 and 4. In Table 3, voltages at nodes 1,2,3,6 and 8 have been omitted because node 1 is a slack bus with voltage reference of $1.06 \mathrm{pu}$, and the others are $P V$ nodes with voltage references $1.045,1.010,1.070$ and $1.090 \mathrm{pu}$ respectively. In the base case a low voltage profile is obtained and the minimum voltage is achieved in node $14(0.92 \mathrm{pu})$, the total system losses for the base case are 117MW (see Table $4)$.

When a shunt or series device is activated the values of its resistance and reactance are set respectively to 0 and $0.06 \mathrm{pu}$. 
In Table 2, all test developed are described. The first column is the code of the case that will be the same in the Tables 3 and 4 . The second column specifies the device location, the shunt connetected devices node, the series connected devices line and the combined devices node and line. Column 3 shows the used control according to the described controls in section III. In column 4, the control references can be observed. Take notice that when using the OPF approach, no control is selected for the device and the OPF target will be the total loss minimization. In columns 5 and 6 the obtained injected voltages can be seen. Finally, columns 7 depicts the injected reactive power when a series or shunt device is used, or the active power exchanged between the series and the shunt devices when an UPFC is employed.

The authors have validated and tested the proposed method by means of a commercial software package PowerFactory by DigSilent. More than 200 cases were tested, activating a maximum of 6 series devices and 6 shunt devices at the same time. In this work, for the sake of simplicity, 22 tests are presented. The first 10 cases correspond to 3 STATCOMs in three different locations and different control, the next 9 cases used SSSCs at 4 different locations and the last 3 cases are simulations with UPFCs.

In case 1 a STATCOM is located at node 14 controlling the voltage at that node with a voltage reference of $1.01 \mathrm{pu}$. To increase the voltage level from 0.92 (base case) to $1.01 \mathrm{pu}$, the device need to inject 43.84MVAr. This reactive power injection causes the increasing of all voltage level profile in the system. The apparent power flowing through the lines is not substantially modified being the highest variation located at lines 17 and 14. In line 17 the apparent power increases due to the STATCOM injection. As a consequence, 
the apparent power through line 14 is reduced. The total amount of losses is reduced in $2 \mathrm{MW}$

In case 2, the voltage in node 10 is controlled by using an STATCOM located at node 14 . In this case the voltage reference at node 10 is $1.0 \mathrm{pu}$ and the amount of injected power by the device is higher than in the previous case (114.6 MVAr). The difference is that in this case the total losses are increased in $3 \mathrm{MW}$ when comparing with the base case.

In case 3 , the STACOM is located at node 4 with an injected voltage reference of $1.0 \mathrm{pu}$, and the device injects $40 \mathrm{MVAr}$.

Case 4 fixes the injected power in node 4 in 100 MVAr. As it was expected, the voltage is increased when comparing to previous case and the total losses are reduced 4MW. Cases 5 and 6 place the device in node 10 controlling the reactive and the active power flow in line 18 respectively. In the case 6 the active power flow through line 18 is reduced to 0 , however, to do that, the device has to inject more than 300 MVAr increasing the whole voltage profile, the apparent power through line 18 and the total losses in 20MW. Obviously, such reference could not be used in case of a constrained power flow, because the voltage at node 10 achieves values of $1.32 \mathrm{pu}$.

In case 7 an impedance reference is used when the STATCOM is located at node 4. Cases 8,9 and 10 are solved with the OPF approach, placing the device at nodes 4,10 and 14 respectively, the constraints were activated and the controls deactivated using just the operational constraints. The voltage constraints in all nodes were set to 0.85 and $1.10 \mathrm{pu}$. In all OPF cases, the total losses were reduced with respect the base case. However, case 10 is quite similar to case 1. Case 8 is similar in terms of losses to case 7 , just 
a difference of $1 \mathrm{MW}$, but the voltage profile of case 8 is higher. The same conclusion could be achieved when comparing the cases 5 and 9, they are similar in terms of losses, but the voltage profile of case 9 is slightly higher.

In cases from 11 to 16, an SSSC has been activated in lines 8, 9, 10 and 13 but with different controls. In all of these cases, except the cases 11 and 12 , when the device was activated in line 9, the total losses has been increased. Even when the OPF approach was tested, the total losses reduction was very low, and in case 17 the total losses increased with respect the base case even when they are much lower than the case 13, when the device was activated in the same line with a fixed control.

Finally, several UPFC were carried out with different node/line combinations. In cases 20,21 and 22, we can observe 3 of the better combinations. In case 20 a loss reduction of $11 \mathrm{MW}$ was achieved. This is a curious case because a the shunt part of the UPFC is connected to the node 6 , which is a $P V$ node, so the device cannot vary the voltage in it. However it absorbs active and reactive power from this node and inject them into the line 13 , thus increasing the voltage of node 13 until the constrained limit of $1.1 \mathrm{pu}$ is achieved. Something similar happens when the UPFC is connected to node 6 and line 11 (case 21). The device cannot rise the voltage at node 6 , however it is able to increase the voltage at node 11, where line 11 is connected, until the limit is reached, in this case, the loss reduction is 17MW. In the last case, the shunt device is connected to node 4 and the series one to line 8 , in this case a reduction in the total losses of $25 \mathrm{MW}$ is obtained with a low voltage profile. In this case, the lower voltage constrain is reached at node 14 . 


\section{Conclusions}

In the present work, the authors have proposed a versatile formulation that allows FACTS models to be embedded in power systems models in a simple and fast way by using the node incidence matrix $(\boldsymbol{\Gamma})$ approach and a rectangular synchronous reference frame. As it was demonstrated, the number and location of devices can be modified without changing the linear core of the problem. As a consequence, the dimension of the problem does not vary, even when the number of active devices does. This fact allows the authors to avoid the tedious tracking routines to search which variables corresponds to which devices (for instance $i_{d 10}^{s h}$, always be the $d$ component of the current in the shunt device connected to node 10 and its position in the solution vector is fixed, if no shunt device is connected to such node, this value will be zero). Finally, all the expressions were referred to the $d q$ reference frame, simplifying the controls modeling and using the same reference frame for the controls and for the rest of power flow equations.

[1] X. Zhang, C. Rehtanz, P. Bikash, Flexible ac transmission systems: Modelling and control, Springer Publishing Company, Incorporated, 2012.

[2] X. Fang, J. Chow, X. Jiang, B. Fardanesh, E. Uzunovic, A. Edris, Sensitivity methods in the dispatch and siting of facts controllers, Power Systems, IEEE Transactions on 24 (2) (2009) 713-720.

[3] W. Shao, V. Vittal, Lp-based opf for corrective facts control to relieve overloads and voltage violations, Power Systems, IEEE Transactions on 21 (4) (2006) 1832-1839. 
[4] X.-P. Zhang, Modelling of the interline power flow controller and the generalised unified power flow controller in newton power flow, Generation, Transmission and Distribution, IEE Proceedings- 150 (3) (2003) $268-274$.

[5] A. L. Ara, A. Kazemi, S. N. Niaki, Modelling of optimal unified power flow controller (oupfc) for optimal steady-state performance of power systems, Energy Conversion and Management 52 (2) (2011) 1325 - 1333.

[6] T. Duong, Y. JianGang, V. Truong, A new method for secured optimal power flow under normal and network contingencies via optimal location of $\{$ TCSC $\}$, International Journal of Electrical Power \& Energy Systems $52(0)(2013) 68-80$.

[7] C. Lehmkoster, Security constrained optimal power flow for an economical operation of facts-devices in liberalized energy markets, Power Delivery, IEEE Transactions on 17 (2) (2002) 603-608.

[8] B. Fardanesh, Optimal utilization, sizing, and steady-state performance comparison of multiconverter vsc-based facts controllers, Power Delivery, IEEE Transactions on 19 (3) (2004) 1321-1327.

[9] G. Yan, G. Hovland, R. Majumder, Z. Dong, Tcsc allocation based on line flow based equations via mixed-integer programming, Power Systems, IEEE Transactions on 22 (4) (2007) 2262-2269.

[10] S. An, J. Condren, T. Gedra, An ideal transformer upfc model, opf first-order sensitivities, and application to screening for optimal upfc locations, Power Systems, IEEE Transactions on 22 (1) (2007) 68-75. 
[11] M. Alomoush, Derivation of upfc dc load flow model with examples of its use in restructured power systems, Power Systems, IEEE Transactions on 18 (3) (Aug.) 1173-1180.

[12] A. Lashkar Ara, A. Kazemi, S. Niaki, Multiobjective optimal location of facts shunt-series controllers for power system operation planning, Power Delivery, IEEE Transactions on 27 (2) (2012) 481-490.

[13] S. Kansal, V. Kumar, B. Tyagi, Optimal placement of different type of dg sources in distribution networks, International Journal of Electrical Power \& Energy Systems 53 (0) (2013) 752 - 760.

[14] M. Gitizadeh, M. Shidpilehvar, M. Mardaneh, A new method for svc placement considering fss limit and svc investment cost, International Journal of Electrical Power \& Energy Systems 53 (0) (2013) 900 - 908.

[15] G. N. Kumar, M. S. Kalavathi, Cat swarm optimization for optimal placement of multiple upfcs in voltage stability enhancement under contingency, International Journal of Electrical Power \& Energy Systems 57 (0) (2014) $97-104$.

[16] X. Wei, J. Chow, B. Fardanesh, A. Edris, A common modeling framework of voltage-sourced converters for load flow, sensitivity, and dispatch analysis, Power Systems, IEEE Transactions on 19 (2) (2004) 934-941.

[17] Y. Zhang, Y. Zhang, B. Wu, J. Zhou, Power injection model of statcom with control and operating limit for power flow and voltage stability analysis, Electric Power Systems Research 76 (12) (2006) 1003 - 1010. 
[18] C. Rakpenthai, S. Premrudeepreechacharn, S. Uatrongjit, Power system with multi-type facts devices states estimation based on predictorcorrector interior point algorithm, International Journal of Electrical Power \& Energy Systems 31 (4) (2009) 160 - 166.

[19] C. Rakpenthai, S. Premrudeepreechacharn, S. Uatrongjit, N. R. Watson, An interior point method for wlav state estimation of power system with upfcs, International Journal of Electrical Power \& Energy Systems 32 (6) (2010) $671-677$.

[20] B. Xu, A. Abur, State estimation of systems with upfcs using the interior point method, Power Systems, IEEE Transactions on 19 (3) (2004) $1635-1641$.

[21] A. Zamora-Cardenas, C. R. Fuerte-Esquivel, State estimation of power systems containing facts controllers, Electric Power Systems Research 81 (4) (2011) $995-1002$.

[22] Y. Xiao, Y. H. Song, Y. Z. Sun, Power flow control approach to power systems with embedded facts devices, Power Systems, IEEE Transactions on 17 (4) (2002) 943-950.

[23] D. Gotham, G. Heydt, Power flow control and power flow studies for systems with facts devices, Power Systems, IEEE Transactions on 13 (1) (1998) 60-65.

[24] N. P. Padhy, M. A. Moamen, Power flow control and solutions with multiple and multi-type facts devices, Electric Power Systems Research 74 (3) (2005) $341-351$. 
[25] A. Vinkovic, R. Mihalic, A current-based model of the static synchronous series compensator (sssc) for newton-raphson power flow, Electric Power Systems Research 78 (10) (2008) 1806 - 1813.

[26] A. Vinkovic, R. Mihalic, A current-based model of an ipfc for newtonraphson power flow, Electric Power Systems Research 79 (8) (2009) 1247 $-1254$.

[27] A. Vinkovic, R. Mihalic, Universal method for the modeling of the 2nd generation $\{$ FACTS $\}$ devices in newtonraphson power flow, International Journal of Electrical Power \& Energy Systems 33 (10) (2011) $1631-1637$.

[28] X.-P. Zhang, Multiterminal voltage-sourced converter-based hvdc models for power flow analysis, Power Systems, IEEE Transactions on 19 (4) (2004) 1877-1884.

[29] S. Bhowmick, B. Das, N. Kumar, An advanced ipfc model to reuse newton power flow codes, Power Systems, IEEE Transactions on 24 (2) (2009) 525-532.

[30] J.-Y. Liu, Y. hua Song, P. Mehta, Strategies for handling upfc constraints in steady-state power flow and voltage control, Power Systems, IEEE Transactions on 15 (2) (2000) 566-571.

[31] R. Palma-Behnke, L. Vargas, J. Perez, J. Nunez, R. Torres, Opf with svc and upfc modeling for longitudinal systems, Power Systems, IEEE Transactions on 19 (4) (2004) 1742-1753. 
[32] M. Pereira, L. Zanetta, A current based model for load flow studies with upfc, Power Systems, IEEE Transactions on 28 (2) (2013) 677-682.

[33] R. Benabid, M. Boudour, M. Abido, Development of a new power injection model with embedded multi-control functions for static synchronous series compensator, Generation, Transmission Distribution, IET 6 (7) (July) 680-692.

[34] S. Nabavi Niaki, R. Iravani, M. Noroozian, Power-flow model and steady-state analysis of the hybrid flow controller, Power Delivery, IEEE Transactions on 23 (4) (2008) 2330-2338.

[35] S. Bhowmick, B. Das, N. Kumar, An indirect upfc model to enhance reusability of newton power-flow codes, Power Delivery, IEEE Transactions on 23 (4) (2008) 2079-2088.

[36] E. Acha, B. Kazemtabrizi, A new statcom model for power flows using the newton raphson method, Power Systems, IEEE Transactions on PP (99) (2013) 1-11.

[37] G. Radman, R. S. Raje, Power flow model/calculation for power systems with multiple facts controllers, Electric Power Systems Research 77 (12) (2007) $1521-1531$.

[38] C. Angeles-Camacho, E. Acha, Phase-domain power flows in the rectangular co-ordinates frame of reference including vsc-based facts controllers, Electric Power Systems Research 78 (3) (2008) 494 - 506.

[39] X.-P. Zhang, Advanced modeling of the multicontrol functional static 
synchronous series compensator (sssc) in newton power flow, Power Systems, IEEE Transactions on 18 (4) (2003) 1410-1416.

[40] X. Jiang, X. Fang, J. Chow, A. Edris, E. Uzunovic, M. Parisi, L. Hopkins, A novel approach for modeling voltage-sourced converter-based facts controllers, Power Delivery, IEEE Transactions on 23 (4) (2008) $2591-2598$

[41] S. Kamel, M. Abdel-Akher, F. Jurado, Improved nr current injection load flow using power mismatch representation of pv bus, International Journal of Electrical Power \& Energy Systems 53 (0) (2013) 64 - 68.

[42] F. Milano, Power System Modelling and Scripting, Springer, 2010.

[43] P. Arboleya, G. Diaz, M. Coto, Unified ac/dc power flow for traction systems: A new concept, Vehicular Technology, IEEE Transactions on PP (99) (2012) 1.

[44] M. Coto, P. Arboleya, C. Gonzalez-Moran, Optimization approach to unified ac/dc power flow applied to traction systems with catenary voltage constraints, International Journal of Electrical Power \& Energy Systems $53(0)(2013) 434-441$.

[45] P. Yan, A. Sekar, Steady-state analysis of power system having multiple facts devices using line-flow-based equations, Generation, Transmission and Distribution, IEE Proceedings- 152 (1) (2005) 31-39.

[46] P. Yan, A. Sekar, Analysis of radial distribution systems with embedded series facts devices using a fast line flow-based algorithm, Power Systems, IEEE Transactions on 20 (4) (2005) 1775-1782. 
[47] I. Cederbaum, Some applications of graph theory to network analysis and synthesis, Circuits and Systems, IEEE Transactions on 31 (1) (1984) $64-68$.

[48] F. Harary, Graph theory and electric networks, Circuit Theory, IRE Transactions on 6 (5) (1959) $95-109$.

[49] Y. Fu, Realization of circuit matrices, Circuit Theory, IEEE Transactions on 12 (4) (1965) $604-607$.

[50] R. Vasquez-Arnez, L. Zanetta, A novel approach for modeling the steady-state vsc-based multiline facts controllers and their operational constraints, Power Delivery, IEEE Transactions on 23 (1) (2008) 457464.

[51] IEEE, Power systems test case archive (Aug. 1999). URL http://www. ee. washington. edu/research/pstca/ 


\section{List of Figures}

Representation of a generic connection between two system nodes with a series real voltage source in the line representing an embedded series FACTS device and a shunt real voltage source at each node, representing an embedded shunt connected FACTS device. . . . . . . . . . . . . . . . . 36

IEEE 14 nodes modified test bus system. . . . . . . . . . . 37 


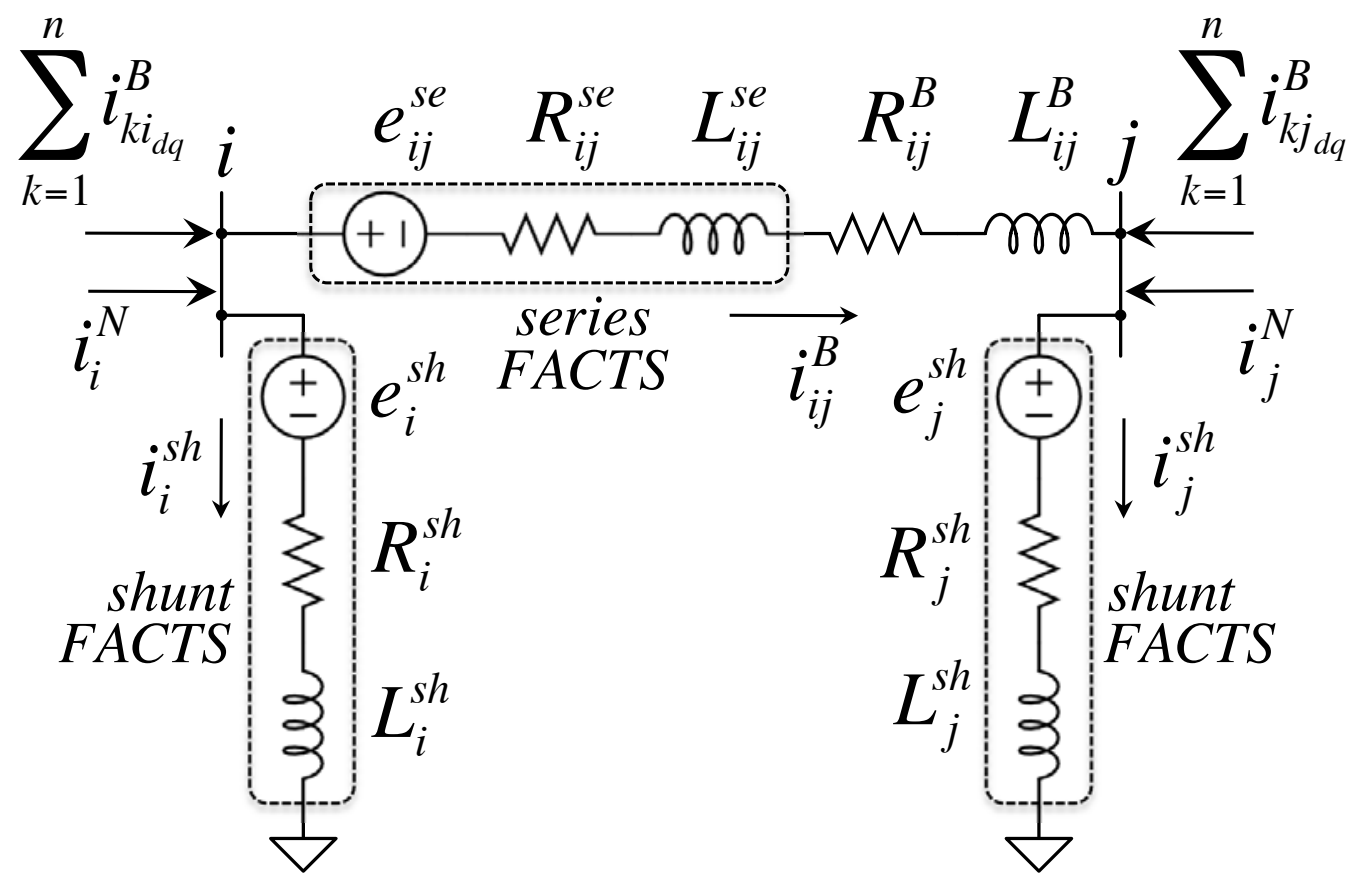

Figure 1: Representation of a generic connection between two system nodes with a series real voltage source in the line representing an embedded series FACTS device and a shunt real voltage source at each node, representing an embedded shunt connected FACTS device. 


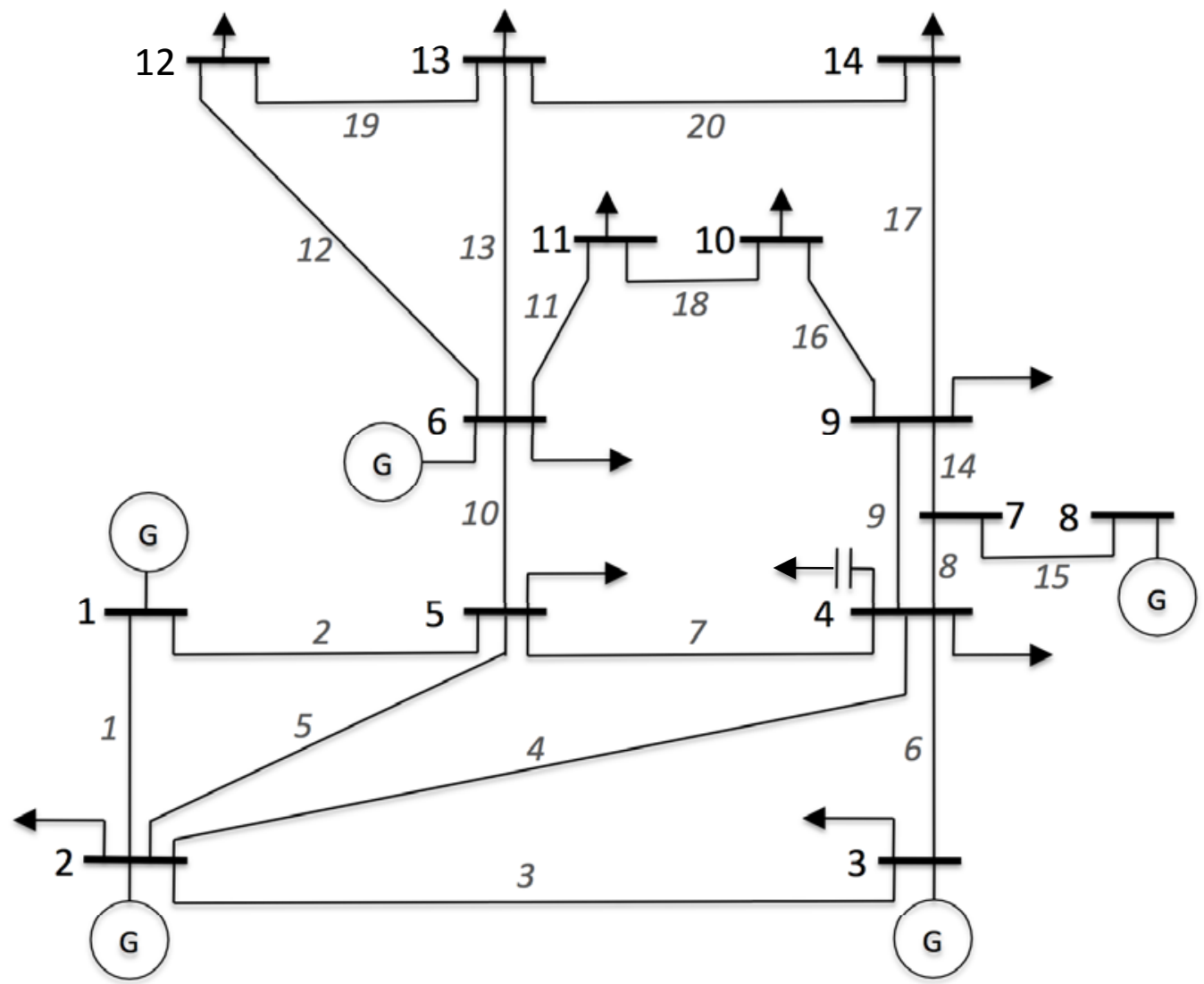

Figure 2: IEEE 14 nodes modified test bus system. 
List of Tables

$616 \quad 1$ Conventional PQ, PV and Slack buses description. . . . . . . . 40

${ }_{617} 2$ Cases description. All voltages are in pu. system and active and reactive powers in MW and MVA respectively. . . . . . . 41

6193 Voltage magnitude in all nodes in per unit system. . . . . . . . 42

${ }_{620} 4$ Aparent powers in all lines in MVA and total system losses in

621

MW. . . . . . . . . . . . . . . . 43 


$\mathbf{M}=\left(\begin{array}{cc|cc|cc|cc|cc|cc}\left(\mathbf{R}^{s e+B}\right) & -\omega\left(\mathbf{L}^{s e+B}\right) & 0 & 0 & 0 & 0 & \mathbf{I} & 0 & 0 & 0 & -\boldsymbol{\Gamma} & 0 \\ \omega\left(\mathbf{L}^{s e+B}\right) & \left(\mathbf{R}^{s e+B}\right) & 0 & 0 & 0 & 0 & 0 & \mathbf{I} & 0 & 0 & 0 & -\boldsymbol{\Gamma} \\ \hline 0 & 0 & \mathbf{R}^{s h} & -\omega \mathbf{L}^{s h} & 0 & 0 & 0 & 0 & \mathbf{I} & 0 & -\mathbf{I} & 0 \\ 0 & 0 & \omega \mathbf{L}^{s h} & \mathbf{R}^{s h} & 0 & 0 & 0 & 0 & 0 & \mathbf{I} & 0 & -\mathbf{I} \\ \hline \boldsymbol{\Gamma}^{T} & 0 & \mathbf{I} & 0 & -\mathbf{I} & 0 & 0 & 0 & 0 & 0 & 0 & 0 \\ 0 & \boldsymbol{\Gamma}^{T} & 0 & \mathbf{I} & 0 & -\mathbf{I} & 0 & 0 & 0 & 0 & 0 & 0\end{array}\right)$




\begin{tabular}{c|c|c|c}
\hline \hline Type & Specified & Unknowns & Equations \\
\hline \multirow{2}{*}{ PQ Bus } & \multirow{2}{*}{$P_{i}, Q_{i}$} & $v_{i d}, v_{i q}, i_{i d}^{N}, i_{i q}^{N}$ & $v_{i d} \cdot i_{i d}^{N}+v_{i q} \cdot i_{i q}^{N}-P_{i}=0$ \\
\cline { 4 - 4 } & & & $v_{i q} \cdot i_{i d}^{N}-v_{i d} \cdot i_{i q}^{N}-Q_{i}=0$ \\
\hline PV Bus & \multirow{2}{*}{$P_{i},\left|v_{i}\right|$} & $v_{i d}, v_{i q}, i_{i d}^{N}, i_{i q}^{N}$ & $v_{i d} \cdot i_{i d}^{N}+v_{i q} \cdot i_{i q}^{N}-P_{i}=0$ \\
\cline { 4 - 4 } & & & $\sqrt{v_{i d}^{2}+v_{i q}^{2}}-\left|v_{i}\right|=0$ \\
Slack Bus & $v_{i d}, v_{i q}$ & $i_{i d}^{N}, i_{i q}^{N}$ & - \\
\cline { 3 - 4 } & & & \\
\hline \hline
\end{tabular}

Table 1: Conventional PQ, PV and Slack buses description. 


\begin{tabular}{c|c|c|l|c|c|c}
\hline \hline Case & node & control & reference & $e_{d}^{s h}$ & $e_{q}^{s h}$ & $Q^{s h}$ \\
\hline 1 & 14 & 1 & $\left|v_{14}\right|=1.01$ & 0.68 & -0.78 & -43.84 \\
2 & 14 & 1 & $\left|v_{10}\right|=1.0$ & 0.74 & -0.92 & -114.16 \\
3 & 4 & 2 & $\left|e^{s h}\right|=1.0$ & 0.86 & -0.51 & -40.00 \\
4 & 4 & 3 & $Q^{s h}=-100$ & 0.91 & -0.54 & -100.00 \\
5 & 10 & 4 & $Q_{18}^{B}=5$ & 0.76 & -0.74 & -49.77 \\
6 & 10 & 5 & $P_{18}^{B}=0$ & 0.97 & -1.12 & -381.17 \\
7 & 4 & 6 & $X^{s h}=-1$ & 0.92 & -0.54 & -114.37 \\
8 & 4 & OPF & Loss min. & 0.97 & -0.57 & -178.87 \\
9 & 10 & OPF & Loss min. & 0.76 & -0.76 & -67.65 \\
10 & 14 & OPF & Loss min. & 0.67 & -0.77 & -36.75 \\
\hline Case & line & control & reference & $e_{d}^{s e}$ & $e_{q}^{s e}$ & $Q^{s e}$ \\
\hline 11 & 9 & 1 & $\left|v_{4}\right|=0.96$ & -0.33 & -0.47 & -61.42 \\
12 & 9 & 1 & $\left|v_{9}\right|=0.96$ & -0.18 & -0.23 & -21.35 \\
13 & 8 & 2 & $\left|e_{14}\right|=1.0$ & 0.65 & -0.76 & 218.33 \\
14 & 10 & 3 & $Q_{10}^{B}=0$ & -0.74 & -1.28 & -442.02 \\
15 & 10 & 4 & $P_{10}^{B}=30$ & 0.78 & 0.58 & 35.56 \\
16 & 13 & 5 & $X_{13}=0.01$ & 0.03 & 0.02 & -3.80 \\
17 & 8 & OPF & Loss min & 0.08 & -0.61 & -101.20 \\
18 & 9 & OPF & Loss min & -0.13 & -0.15 & -11.95 \\
19 & 13 & OPF & Loss min & -0.04 & -0.01 & -2.19 \\
\hline Case & node/line & contr ol & reference & $e^{s e}$ & $e^{s h}$ & $P^{s h}$ \\
\hline 20 & $6 / 13$ & OPF & Loss min & 0.45 & 0.98 & 35.62 \\
21 & $6 / 11$ & OPF & Loss min & 0.97 & 1.01 & 68.15 \\
22 & $4 / 8$ & OPF & Loss min & 1.36 & 0.91 & 53.54 \\
\hline \hline
\end{tabular}

Table 2: Cases description. All voltages are in pu. system and active and reactive powers in MW and MVA respectively. 


\begin{tabular}{c|c|c|c|c|c|c|c|c|c}
\hline \hline Case & $v_{4}$ & $v_{5}$ & $v_{7}$ & $v_{9}$ & $v_{10}$ & $v_{11}$ & $v_{12}$ & $v_{13}$ & $v_{14}$ \\
\hline Base & 0.96 & 0.96 & 0.98 & 0.94 & 0.94 & 1.00 & 1.02 & 1.00 & 0.92 \\
\hline 1 & 0.96 & 0.97 & 1.00 & 0.97 & 0.97 & 1.01 & 1.04 & 1.03 & 1.01 \\
2 & 0.97 & 0.97 & 1.02 & 1.01 & 1.00 & 1.03 & 1.05 & 1.05 & 1.13 \\
3 & 0.98 & 0.98 & 0.99 & 0.95 & 0.95 & 1.00 & 1.02 & 1.00 & 0.92 \\
4 & 1.00 & 0.99 & 1.00 & 0.96 & 0.96 & 1.00 & 1.03 & 1.01 & 0.93 \\
5 & 0.97 & 0.97 & 1.01 & 0.99 & 1.01 & 1.03 & 1.03 & 1.01 & 0.95 \\
6 & 0.99 & 0.99 & 1.10 & 1.18 & 1.32 & 1.19 & 1.04 & 1.04 & 1.08 \\
7 & 1.01 & 0.99 & 1.01 & 0.96 & 0.96 & 1.00 & 1.03 & 1.01 & 0.93 \\
8 & 1.03 & 1.01 & 1.02 & 0.97 & 0.97 & 1.01 & 1.03 & 1.01 & 0.94 \\
9 & 0.97 & 0.97 & 1.01 & 1.00 & 1.04 & 1.04 & 1.03 & 1.01 & 0.96 \\
10 & 0.96 & 0.97 & 1.00 & 0.97 & 0.96 & 1.01 & 1.03 & 1.02 & 1.00 \\
\hline 11 & 0.96 & 0.97 & 1.00 & 0.96 & 0.96 & 1.01 & 1.02 & 1.01 & 0.93 \\
12 & 0.96 & 0.97 & 0.99 & 0.95 & 0.95 & 1.00 & 1.02 & 1.01 & 0.92 \\
13 & 0.80 & 0.84 & 0.43 & 0.50 & 0.56 & 0.80 & 0.99 & 0.93 & 0.60 \\
14 & 0.94 & 0.92 & 0.93 & 0.83 & 0.82 & 0.91 & 1.01 & 0.96 & 0.81 \\
15 & 0.89 & 0.91 & 0.92 & 0.87 & 0.87 & 0.95 & 1.01 & 0.99 & 0.85 \\
16 & 0.51 & 0.44 & 0.57 & 0.46 & 0.52 & 0.77 & 0.97 & 0.90 & 0.53 \\
17 & 1.00 & 0.99 & 0.90 & 0.90 & 0.91 & 0.98 & 1.02 & 1.00 & 0.88 \\
18 & 0.96 & 0.97 & 0.99 & 0.95 & 0.95 & 1.00 & 1.02 & 1.00 & 0.92 \\
19 & 0.96 & 0.96 & 0.98 & 0.94 & 0.94 & 1.00 & 1.02 & 1.01 & 0.92 \\
\hline 20 & 0.95 & 0.96 & 0.99 & 0.95 & 0.95 & 1.00 & 1.09 & 1.10 & 0.96 \\
21 & 0.94 & 0.96 & 0.98 & 0.95 & 0.97 & 1.10 & 1.02 & 1.00 & 0.91 \\
22 & 0.97 & 0.98 & 0.91 & 0.87 & 0.87 & 0.95 & 1.01 & 0.99 & 0.85 \\
\hline \hline
\end{tabular}

Table 3: Voltage magnitude in all nodes in per unit system. 


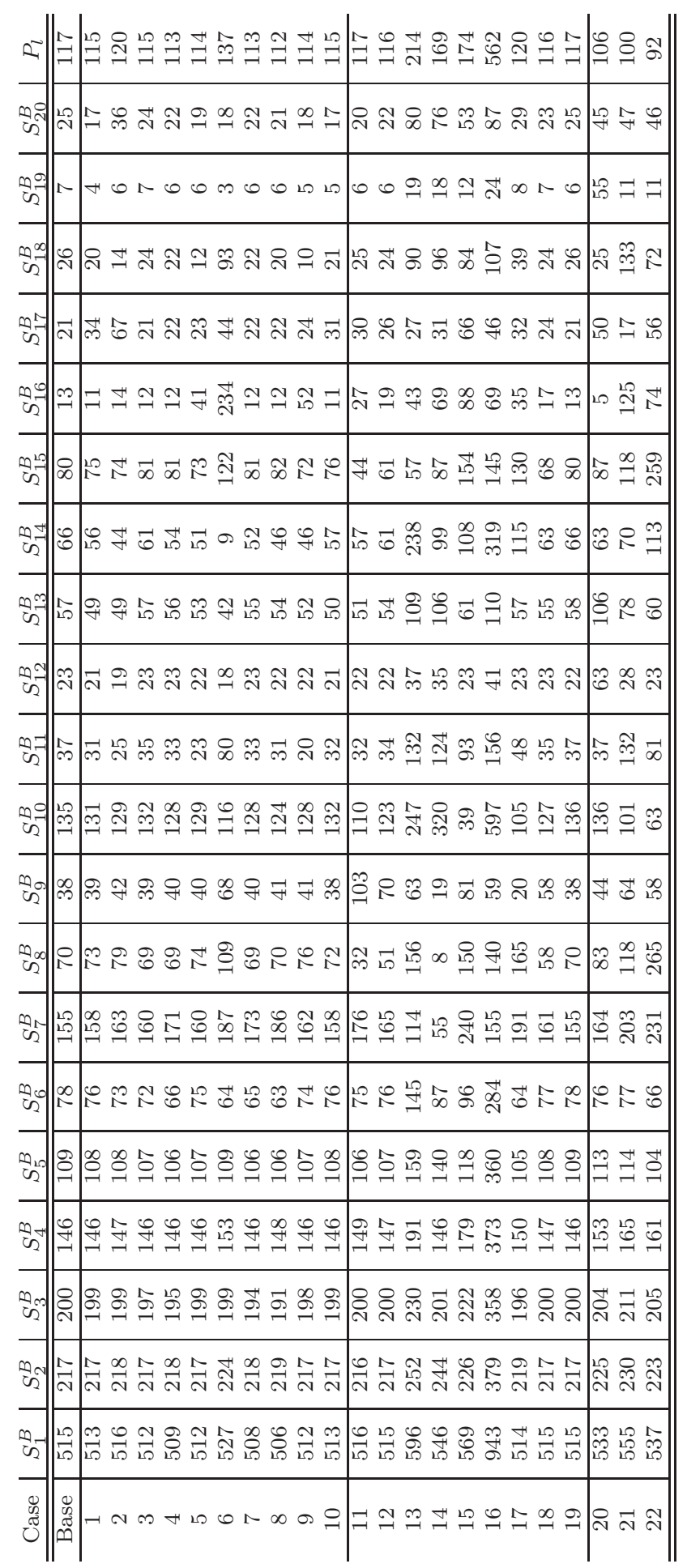

Table 4: Aparent powers in all lines in MVA and total system losses in MW. 